\title{
Fe XIX observations of active region brightenings in the corona
}

\author{
T. J. Wang ${ }^{\star}$, D. E. Innes, and S. K. Solanki
}

Max-Planck Institut für Sonnensystemforschung, 37191 Katlenburg-Lindau, Germany ${ }^{\star \star}$
e-mail: wangt j@mps .mpg. de

Received 2 March 2006 / Accepted 15 May 2006

\section{ABSTRACT}

\begin{abstract}
Small flarelike brightenings seen in the hot flare line, Fe XIX, by the spectrometer SUMER on SOHO are analysed. We observe active region coronae about $30 \mathrm{Mm}$ off the limb of the Sun for a period of several days. Brightenings are observed with a frequency 3-14 per hour and their lifetimes range from 5-150 min, with an average of about $25 \mathrm{~min}$. The measured size of the events along the spectrometer slit range from 2-67 Mm, but most are around $7 \mathrm{Mm}$. Like soft X-ray active region transient brightenings, they range in estimated thermal energy from $10^{26}$ to $10^{29} \mathrm{erg}$ with a power law index of 1.7 to 1.8 , beyond $10^{27} \mathrm{erg}$. We conclude that they are the coronal parts of loops heated to $>6 \mathrm{MK}$ by soft X-ray microflares.
\end{abstract}

Key words. Sun: activity - Sun: corona - Sun: flares - Sun: UV radiation - Sun: X-rays, gamma rays

\section{Introduction}

Active region transient brightenings (ARTBs) are very frequent soft X-ray brightenings seen in active regions (Shimizu et al. 1992; Shimizu 1995). Their frequency, 1-20 events per hour, correlates with the regions' total soft X-ray flux and they are seen as an important link in understanding the heating of the corona. The brightest produce hard X-ray and nonthermal radio emission bursts at the start of the events (Gary et al. 1997; Nitta 1997; Qiu et al. 2004). They are therefore low energy subflares and have been used to extend the flare event statistics down to a few times $10^{26} \mathrm{erg}$, the microflare energy range. The frequency distribution for both the soft and hard X-ray flarelike events is a power-law function with an index of 1.6-1.8 (Lin et al. 1984; Crosby et al. 1993; Shimizu 1995; Qiu et al. 2004) across six orders of magnitude in energy. Thus it appears that the total power input to the corona decreases with event energy (Hudson 1991) and microflares are insignificant to heating. Nevertheless they do occur about a million times more frequently than subflares and are responsible for the regions' relentless activity.

The aim here is to use high resolution spectral observations of events seen about $30 \mathrm{Mm}$ off the limb of the Sun to investigate microflares in the corona. Previous studies of microflares have concentrated on active regions seen on the disk (Shimizu et al. 1992; Berghmans et al. 2001). Most events cause brightening along loops. In about half of the events the whole loop is seen to brighten simultaneously. The other half brightens from a footpoint (single loop) or a low-lying contact point (multiple loops), suggesting heated chromospheric material. Most of these loops will not reach heights of $30 \mathrm{Mm}$, but the microflare rate is so high that it only requires a small percentage in tall loops to give significant statistics for this study. In our initial analysis of the regions studied here, we counted an Fe XIX event rate of 2 per hour (Innes \& Wang 2004) which is consistent with estimates by Shimizu et al. (1994) of microflaring loop sizes and rates.

^ Current address: Department of Physics, Montana State University, Bozeman, MT 59717-3840

$\star \star$ Previously known as MPI für Aeronomie.
In this paper we discuss the properties of microflares seen in the SUMER Fe XIX emission line. We make comparisons with the soft X-ray events. The advantage of using the Fe XIX SUMER spectral line is its high sensitivity to small events, the continuous time coverage and the additional Doppler shift information. We make a statistical study of the physical properties of microflare loops seen in Fe XIX and calculate frequency distributions of their peak intensities and energies.

\section{Observations of flarelike brightenings: data description}

The data sets analysed are SUMER spectral observations of active region coronae made in sit-and-stare mode over a period of several days. Two different regions on the east limb are studied. The slit positions are shown in Fig. 1. The first region was observed with a cadence of $90 \mathrm{~s}$ using the $300^{\prime \prime} \times 4^{\prime \prime}$ slit from 16-20 Sep. 2000. During this time two neighbouring active regions (AR 9167 and AR 9169) rotated onto the disk (see Fig. 1b). Five spectral lines, Fe XIX $\lambda 1118$ (6.3 MK), Ca XV $\lambda 1098$ and $\lambda 555 \times 2(3.5 \mathrm{MK})$, Ca XIII $\lambda 1134$ (2.2 MK), and Si III $\lambda 1113(0.06 \mathrm{MK})$ were transmitted, with a $2.2 \AA$ wide window for each line. The observing sequence was interrupted every $12.6 \mathrm{~h}$ by a full spectrum (800-1600 A) scan of $3.4 \mathrm{~h}$. Observations of the second region, in the south-east, were made from 25-30 Sep. 2000, with a cadence of $162.5 \mathrm{~s}$ and the $300^{\prime \prime} \times$ 4 " slit. The transmitted spectral window was 1097-1119 ̊. It contains a number of lines formed in the temperature range 0.01-6.3 MK, e.g. Fe XIX $\lambda 1118$, Ca XV $\lambda 1098$, Al XI $\lambda 550 \times 2$ (1.6 MK), Ca X $\lambda 557 \times 2(0.7 \mathrm{MK}), \mathrm{Ne}$ VI $\lambda 558 \times 2(0.3 \mathrm{MK})$, and Si III $\lambda 1113$ (see also Feldman et al. 2003). These observations were also interrupted every so often by a full spectrum scan of 3-4 h. In this paper we concentrate on the Fe XIX observations. These are the signatures of microflares closest to soft X-ray data and allow a direct comparison with the analyses of Shimizu et al. (1992, 1994) and Shimizu (1995). The other lines can be used to diagnose the cooling plasma (Innes \& Wang 2004) and prominence material. They will be discussed in subsequent papers.

The soft X-ray fluxes from the GOES full Sun monitors and SXT (Tsuneta et al. 1991) on Yohkoh are used for identifying the 

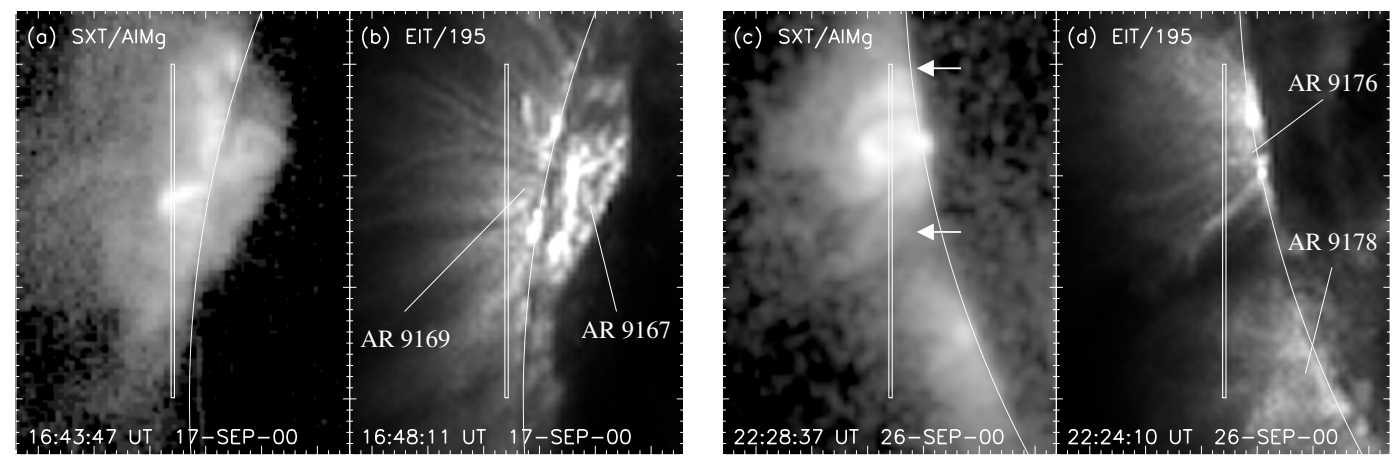

Fig. 1. a) Image of loop systems in AR 9167 and AR 9169 observed with the SXT AlMgMn-sandwich filter. The off limb position of the SUMER slit is marked as a narrow box. b) The same region observed with EIT $195 \AA$ A filter. c) and d) Same as a) and b), but for AR 9176 and AR 9178. The field of view for all images is $300^{\prime \prime} \times 400^{\prime \prime}$.

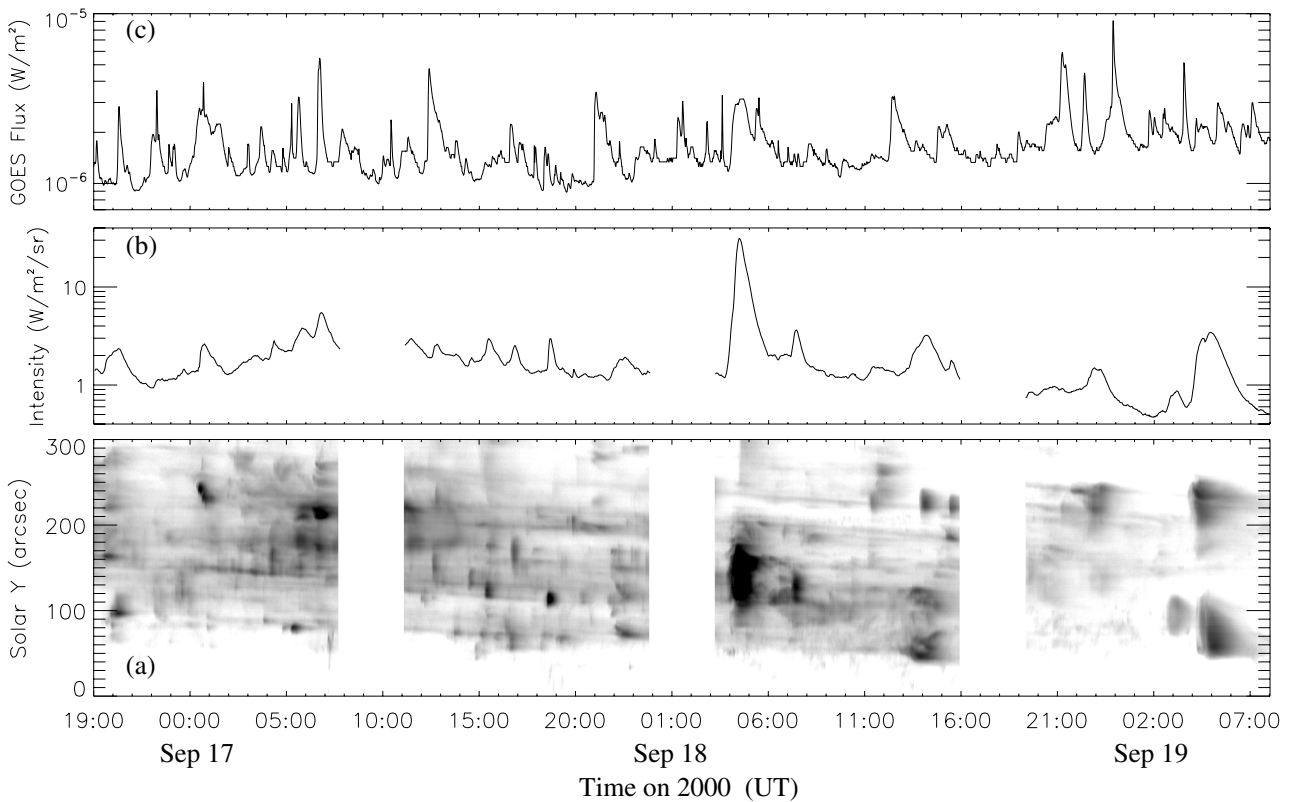

Fig. 2. Fe XIX (SUMER) observations of AR9169 a) Time series of Fe XIX line integrated intensity b) Light curve of Fe XIX integrated over the whole slit c) GOES 1-8 ̊̊ flux.

microflares. GOES gives uninterrupted values of the total soft X-ray flux (0.5-4 $\AA$ and $1-8 \AA$ ) from the entire Sun. Although there were a couple of other active regions on the disk at the time, it is possible to detect individual small, low C-class flares above the background. Thus if the Fe XIX is produced by flares, they can be identified in GOES by comparing light curves of the two emissions. SXT observations of the SUMER target active regions (e.g. AR 9167 and AR 9176) were made at very high cadence, but only for short periods $(<30 \mathrm{~min})$. Some of these SXT images have already been used for seeing the structure of the Fe XIX emitting plasma (Wang et al. 2003a; Innes \& Wang 2004). Here we use the time series of the intensities in the AlMgMn filter to assess how well the Fe XIX observations reflect the coronal soft X-ray emission and thus the coronal microflares. In order to compare SXT with the SUMER observations, the scale of the SXT images is increased by a factor of about $1 \%$ to account for the different orbits.

\section{Results: microflare structure}

\subsection{Fe XIX transient brightenings}

Data from the two series of Fe XIX observations are shown in Figs. 2a and 3a. There appears to be a trend from small, frequent events when the active region is closest to the limb to large, well separated ones. This makes sense because as the active region rotates onto the disk and further away from the SUMER slit, there are fewer and fewer loops that reach the slit and those that do are inevitably bigger. In each series there are events that contradict the trend. The bright emission seen around UT 4:00 on Sep. 18 was due to a large C3.1 flare. In the second series, the large emission patch at UT 13:00 on Sep. 26 was from the southern active region (AR9178) behind the limb.

\subsection{Comparison of FeXIX brightenings with GOES data}

The light curves of the Fe XIX intensity integrated over the whole slit and the GOES 1-8 $\AA$ flux are plotted in Figs. 2b,c and 3b,c above their corresponding Fe XIX time series images. We identified about $53 \mathrm{Fe}$ XIX enhancements from the light curves on 16-19 Sep. and 25-28 Sep., and find that nearly 40 events coincide in time with the GOES C-class flares. A detail of events between 12:30 and 17:30 on Sep. 27 is shown in Figs. 4a,b. Seven brightenings show up as six peaks in the Fe XIX light curve in good agreement with GOES X-ray C1-C3 flux enhancements. 


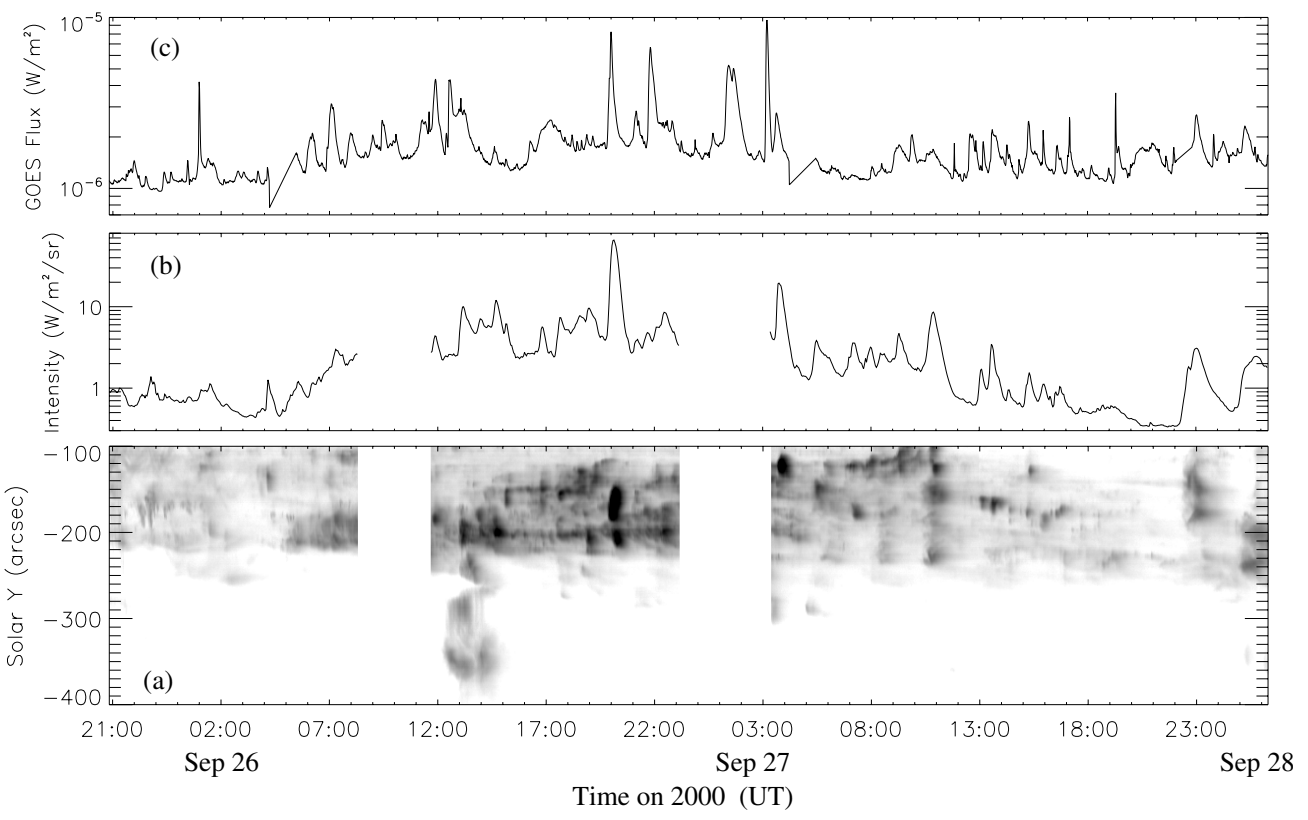

Fig. 3. Fe XIX (SUMER) observations of AR9176 a) Time series of Fe XIX line integrated intensity b) Light curve of Fe XIX integrated over the whole slit c) GOES 1-8 ̊̊ flux.

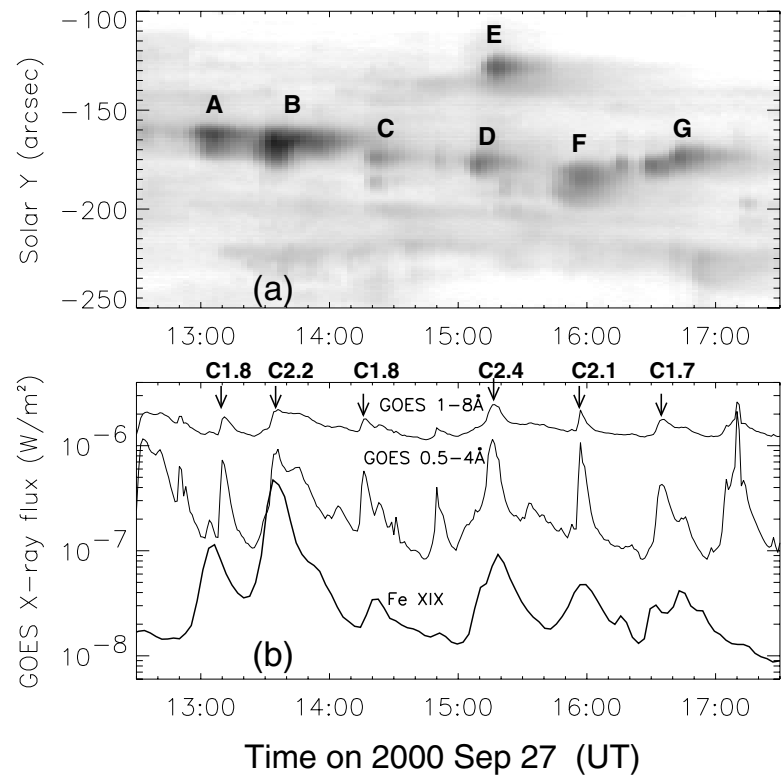

Fig. 4. a) Intensity time series of the Fe XIX line detected at the upper part of the slit shown in Fig. 1c (marked by two arrows). b) Light curve of the Fe XIX line integrated along the slit in arbitrary units (lower line) and light curves of GOES full-sun soft X-ray flux through $1-8 \AA$ and $0.5-4 \AA$. The flux of GOES $0.5-4 \AA$ is multiplied by a factor of 10 .

\subsection{Comparison with SXT data}

Having identified the SUMER Fe XIX brightenings as small flares observed in GOES X-ray flux, we now look at SXT observations. The SXT emission at the position of the SUMER slit is extracted and compared to that in Fe XIX (Fig. 5). We find that all major events (marked 1, 2, P1, P2 and P3) seen in Fe XIX are also detected with SXT. We notice that the lifetimes of these Fe XIX brightenings are typically longer than the SXT observing duration ( $\sim 30 \mathrm{~min})$. Fig. $5 \mathrm{c}$ is a comparison of light curves of the Fe XIX and SXT data, showing that they are well

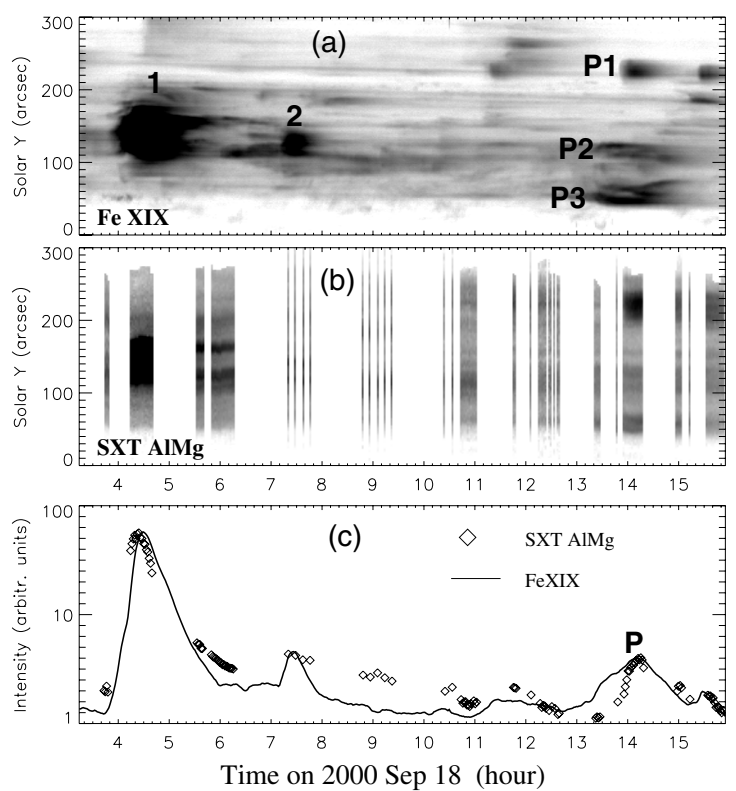

Fig. 5. a) Intensity time series of the Fe XIX line detected at the slit position shown in Figs. 1a and b. b) Same as a) but for the SXT AlMgMnsandwich filter. c) Light curves of the Fe XIX line and the SXT AlMgMn filter integrated over the slit.

coincident in time. The comparison indicates that the emission seen by SUMER in the Fe XIX line and the SXT brightenings are signatures of the same phenomena. The long, nearly uninterrupted SUMER time series are better suited to carrying out a statistical study of coronal brightenings.

\section{Statistical analysis}

\subsection{Identification of brightening events}

The time series (e.g. Figs. 2a and 3a) show many brightenings and a large range in both intensity and lifetime. Some events 


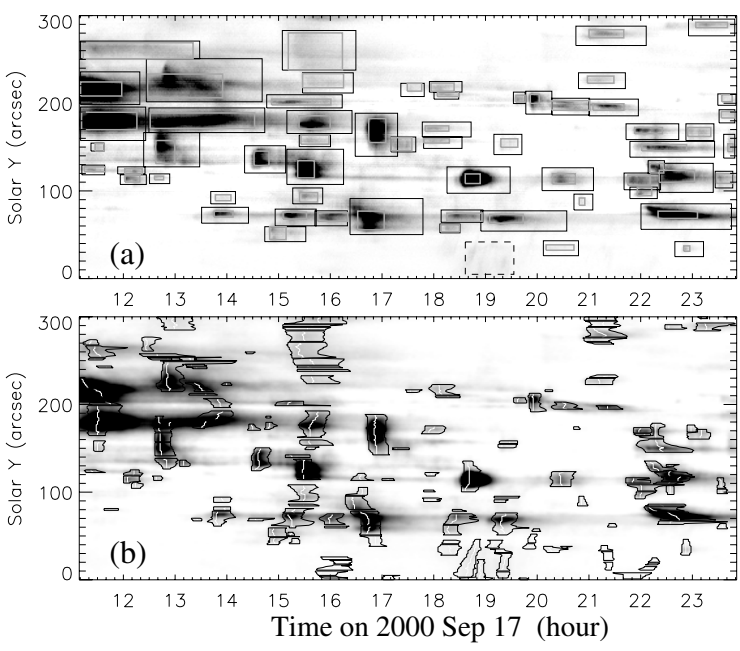

Fig. 6. Examples of brightening identification in Fe XIX space-time diagrams, with a) visual inspection and b) automatic search. In a) the black boxes roughly delineate the position and time of events, and the enclosed grey boxes the FWHM of the intensity peaks. In b) each irregular quadrilateral surrounds an event identified automatically, whose start and end boundaries are determined by the FWHM of temporal peaks of intensity at each spatial pixel and between whose upper and lower boundaries those pixels fulfill the time coincidence criterion. The white curves mark the peak times of intensity in an event. The time series of the Fe XIX intensity shown here is the same as the second block of Fig. 2a, but with the background removed.

contain multiple peaks both along the slit and in time and we have to decide whether they are single, big events or several small independent, partly overlapping ones (i.e. we need to define criteria to help pick out events). Visual inspection is an easy way but cannot deal with these questions objectively. Automatic search is, in principle, superior for a statistical study, but it often requires complicated fine tuning. Since both methods have advantages and disadvantages, we employ both.

In order to easily discern brightenings in time series of the Fe XIX intensity, we first remove the background emission. The background intensity is calculated with an iterative scheme similar to that used by Brković et al. (2001). The background for each spatial pixel along the slit is computed independently. First the time-averaged intensity observed by the pixel is computed. Then events are identified using the criterion that events are brighter than 1.5 times the pixel average. Events are then removed from the computation of pixel average, giving a lower value for the average. This leads to new events which are subsequently also removed. The process is repeated until no more events are found. The background for that pixel is then the final average nonflaring intensity. Figure 6 shows the second section of Fig. 2a, with the background subtracted.

\subsubsection{Visual inspection}

We select brightenings manually one by one and select weak ones by increasing the image contrast. The selected brightenings are enclosed by black boxes in Fig. 6a. Note that the event at UT 19:00 inside the dashed-line box is excluded, because this emission is due to a $\mathrm{PV}$ line blend in the blue wing of the Fe XIX line, about $0.1 \AA$ away from the line center. The PV is difficult to separate from the Fe XIX emission because Fe XIX has a line width of $0.14 \AA$ in the decay phase of brightenings. Fortunately, we can determine whether the $\mathrm{PV}$ is contaminating the Fe XIX emission because P V correlates with the cool transition region line, Si III, that we observed simultaneously in a separate spectral window. We find that such pseudo-events are generally weak and much less frequent than Fe XIX events, so that they hardly affect the statistical result obtained by our automatic search, which does not recognize all blended emissions. We define the duration of an event as the FWHM of the light curve integrated over the selected region along the slit. The extension of an event along the slit is the FWHM of the brightening integrated in time (see the grey boxes shown in Fig. 6a). In some cases we find that the FWHM criterion cannot be fulfilled in the selected region of a brightening. For example, if events are so close together that the rise part of the light curve of the second event is higher than the half maximum peak, we take the minimum between the events as the start time of the second event. Likewise, the criterion for determining the end time of events and extensions along the slit are adjusted where necessary. Furthermore, all selected events should fulfil the condition that the peak intensity is at least $1 \sigma$ above the noise level. For the two data sets with a total observing duration of 96.6 hours, a total of 299 events are identified.

\subsubsection{Automatic search}

After the removal of the background emission, we identify flarelike events in three steps. In the first step, we mark events in the light curves of each pixel using the criterion that enhancements of intensity relative to the minimum intensities preceding and following the event are above a given threshold (i.e., $I_{\text {dif }} \geq \mathcal{N} \sigma$ ). In the second step, we group together neighbouring pixels with simultaneous brightenings identified in the first step. Here, simultaneous means when the time difference between the peak intensities at two neighbouring pixels is within a given threshold (i.e., $\Delta T_{p} \leq \mathcal{N} \Delta t$, where $\Delta t$ is the time cadence). In the third step, we separate event groups. If an event after the pixel grouping consists of at least 4 pixels along the slit and the light curve of the group contains several peaks, we divide this into individual peaks, making sure that the smallest events are at least $L_{y} \geq \mathcal{N}$ pixels along the slit.

Figure $6 \mathrm{~b}$ shows an example of events identified by the automatic search fulfilling the criteria of $I_{\text {dif }} \geq 1 \sigma, \Delta T_{p} \leq 3 \Delta t$, and $L_{y} \geq 3$ pixels. Compared to the the visual inspection method, the automatic procedure finds a larger number of small events because the bigger events are split and sometimes small brightenings that we ignore by eye satisfy our automatic criteria. For the two data sets of Sep. 16-19 and Sep. 25-28, the total number of events which satisfy $\Delta T_{p} \leq 3 \Delta t, L_{y} \geq 3$ pixels and $I_{\mathrm{dif}} \geq 1 \sigma$, $2 \sigma$, and $3 \sigma$ is 1334,863 , and 633 , respectively. We find that the total number of events is less sensitive to the conditions of $\Delta T_{p} \leq \mathcal{N} \Delta t$ or $L_{y} \geq \mathcal{N}$ pixels, compared to $I_{\mathrm{dif}} \geq \mathcal{N} \sigma$. For example, for the case of $\Delta T_{p} \leq 1 \Delta t, 2 \Delta t, \ldots, 5 \Delta t$ (with $I_{\text {dif }} \geq 2 \sigma$ and $L_{y} \geq 3$ pixels), the total number of events vary from 998 to 818 . For $L_{y} \geq 2,3,4$ pixels (with $I_{\text {dif }} \geq 2 \sigma$ and $\Delta T_{p} \leq 3 \Delta t$ ), the total number of events vary from 1027 to 746 .

\subsection{Physical condition of brightenings}

The physical parameters of brightenings found by visual inspection (299 events) and with the automatic search (1334 events) are compared in Table 1 . The automatic search criteria were $I_{\text {dif }} \geq 1 \sigma, \Delta T_{p} \leq 3 \Delta t$, and $L_{y} \geq 3$ pixels. We define the duration of each event as the average of the durations for all spatial pixels in the event, where the duration at a single pixel is 
Table 1. Physical parameters of flarelike brightenings ${ }^{a}$.

\begin{tabular}{lcccc}
\hline \hline \multirow{2}{*}{ Parameters } & \multicolumn{2}{c}{ Visual } & \multicolumn{2}{c}{ Automatic $^{\mathrm{b}}$} \\
& Average & Range & Average & Range \\
\hline$T_{\text {dur }}(\mathrm{min})$ & $33 \pm 20$ & $6-157$ & $22 \pm 13$ & $5-84$ \\
$L_{y}(\mathrm{Mm})$ & $9 \pm 5$ & $3-30$ & $7 \pm 6$ & $2-67$ \\
$I_{\text {peak }}\left(\mathrm{W} / \mathrm{m}^{2} / \mathrm{sr}\right)$ & $0.06 \pm 0.20$ & $0.001-2.4$ & $0.03 \pm 0.12$ & $9 \times 10^{-5}-2.2$ \\
$E_{\text {rad }}(\mathrm{erg})$ & $2.2 \pm 8.0 \times 10^{22}$ & $1.1 \times 10^{20}-1.2 \times 10^{24}$ & $5.4 \pm 27 \times 10^{21}$ & $2.8 \times 10^{18}-5.1 \times 10^{23}$ \\
$\mathrm{EM}\left(\mathrm{cm}^{-5}\right)$ & $0.7 \pm 2.3 \times 10^{28}$ & $1.6 \times 10^{26}-2.7 \times 10^{29}$ & $0.3 \pm 1.4 \times 10^{28}$ & $10^{25}-2.5 \times 10^{29}$ \\
$n_{\mathrm{e}}\left(\mathrm{cm}^{-3}\right)$ & $2.3 \pm 2.3 \times 10^{9}$ & $4.8 \times 10^{8}-2 \times 10^{10}$ & $1.3 \pm 1.6 \times 10^{9}$ & $1.2 \times 10^{8}-1.9 \times 10^{10}$ \\
$E_{\text {therm }}(\mathrm{erg})$ & $8.6 \pm 12 \times 10^{27}$ & $7.1 \times 10^{26}-1.5 \times 10^{29}$ & $4.6 \pm 11 \times 10^{27}$ & $10^{26}-1.5 \times 10^{29}$ \\
\hline
\end{tabular}

${ }^{a} T_{\text {dur }}$ is the duration of events; $L_{y}$ the brightening extension along the slit; $I_{\text {peak }}$ the peak intensity; $E_{\text {rad }}$ the integrated energy (fluence) observed in the Fe XIX line; EM the emission measure which is calculated by $I_{\text {peak }} / G_{\max }$, where $G_{\max }$ is the maximum emissivity of the Fe XIX line; $n_{\mathrm{e}}$ the electron density calculated assuming a constant line-of-sight depth of $7 \mathrm{Mm} ; E_{\text {therm }}$ the thermal energy calculated assuming that the temperature of brightening loops is $6.3 \mathrm{MK}$, the loop length is 3 times $L_{y}$ and the loop width is $7 \mathrm{Mm}$.

${ }^{b}$ satisfying $I_{\mathrm{dif}} \geq 1 \sigma, \Delta T_{p} \leq 3 \Delta t$, and $L_{y} \geq 3$ pixels.
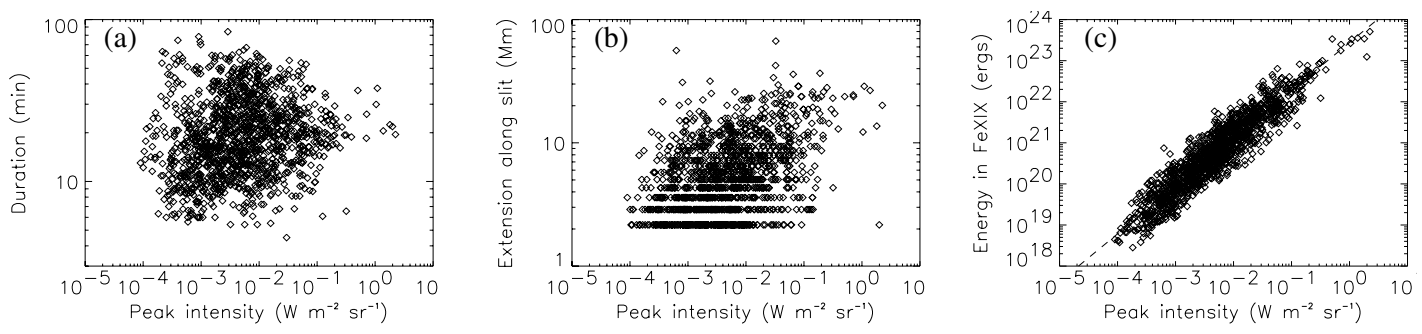

Fig. 7. Distribution of a) the duration, b) the extension along the slit, $\mathbf{c}$ ) the integrated energy of the Fe XIX events as a function of the peak intensity. In c) the dashed line represents a linear fit, obtained with the automatic search (see text). The discrete steps below $6 \mathrm{Mm}$ in Fig. $7 \mathrm{~b}$ are due to pixel digitization (e.g., the lowest row of data points is 3 pixels along the slit).

the FWHM of the intensity enhancement relative to its preceding minimum. The extension of each event along the slit is the scale of all grouped pixels making the event. The other directly measured parameters are the Fe XIX peak intensity, and the total radiated energy observed in the Fe XIX line. As one would expect the parameters deduced from the two search methods are similar. The main difference is that the automatic search finds more small events. In the table, the errors in quantities such as intensity and energy are larger than the average, reflecting the fact that the distributions are non-Gaussian. In the next section we show that they follow a power-law.

Figures 7a,b show distributions of the duration and spatial extension of brightenings with respect to the Fe XIX peak intensity for the automatic search. The durations of brightenings are distributed in a wide range from 5 to $84 \mathrm{~min}$, with a mean of $22 \mathrm{~min}$. The shortest time is three times the cadence. Higher cadence observations may detect shorter bursts because Berghmans et al. (2001) found 1 min microflares in SXT images taken with a cadence $8 \mathrm{~s}$. The short lifetimes were, however, always associated with small events, so they would probably not be seen at $30 \mathrm{Mm}$ off the limb. The longer times are consistent with soft X-ray flare observations (Drake 1971; Veronig et al. 2002), but of course not with SXT microflares because of the short, $30 \mathrm{~min}$, SXT observing periods. The extensions along the slit reflect, if anything, the loop width at the height of the SUMER slit. The range $2-67 \mathrm{Mm}$ is too big to believe that this is always the case. The longer extensions are possibly from loop tops or multiple loops, but without images it is not possible to be sure. In the few cases where we have images, we find that longer loops have a longer extension along the slit. In Sect. 4.3.2 where we compute the event thermal energy, we assume a linear relationship between extension and loop length and for typical loop width we will use the average extension along the slit, $7 \mathrm{Mm}$.
The Fe XIX radiated energy (fluence) of an event is defined as the total flux integral in each quadrilateral outlining the event (see Fig. 6). The distribution of the Fe XIX fluence versus the peak intensity is shown in Fig. 7c for the automatic search. The corresponding linear Pearson correlation coefficient is 0.89 . From the linear fit in log-log space, we obtain the relation $E_{\mathrm{rad}} \sim\left(I_{\text {peak }}\right)^{1.16}$. For almost 50000 soft X-ray flares observed by GOES, Veronig et al. (2002) obtained the relations $F \sim\left(F_{\mathrm{P}}\right)^{1.10}$ where $F$ is the fluence of flares and $F_{\mathrm{P}}$ the peak flux. We find that the results obtained for Fe XIX brightenings are very similar to those for GOES soft X-ray flares.

The peak intensity of Fe XIX events ranges from $10^{-4}$ to $2.2 \mathrm{~W} \mathrm{~m}^{-2} \mathrm{sr}^{-1}$. Under the assumption that the temperature at peak intensity is at a value of $6.3 \mathrm{MK}$, i.e., the temperature where the emissivity reaches the maximum $\left(G_{\max }\right)$, we can estimate the emission measure by $E M=I_{\text {peak }} / G_{\text {max }}$, where $G_{\max }=8.8 \times 10^{-27} \mathrm{erg} \mathrm{cm}^{3} \mathrm{~s}^{-1} \mathrm{sr}^{-1}$ calculated using CHIANTI (e.g. Young et al. 2003). We obtain emission measures in the range $10^{25}-10^{29} \mathrm{~cm}^{-5}$. Assuming the plasma depth along the line of sight to be $7 \mathrm{Mm}$ (the average extension along the slit) and a filling factor of unity, we estimate the electron density to be $10^{8}-2 \times 10^{10} \mathrm{~cm}^{-3}$. The upper limit of our estimate is comparable to that of SXT ARTBs (Shimizu 1995), whereas the lower limit is smaller by an order of magnitude.

\subsection{Frequency of events}

Many previous studies have shown that frequency distributions of peak flux, fluence and total energy for flares and microflares can be represented above a sensitivity threshold by power laws of the form

$\mathrm{d} N=A x^{-\alpha} \mathrm{d} x$ 
Table 2. The power-law index of frequency functions of the peak intensity and the energy of events.

\begin{tabular}{|c|c|c|c|c|}
\hline Condition & $\alpha$ (Peak Intensity) & $\alpha$ (Radiated Energy) & $\alpha$ (Thermal Energy) $)_{\text {fix }}^{c}$ & $\alpha$ (Thermal Energy $)_{\text {random }}^{\mathrm{c}}$ \\
\hline Visual $^{a}$ & $1.60 \pm 0.08$ & $1.79 \pm 0.13$ & $2.05 \pm 0.15$ & $1.88-2.13$ \\
\hline \multicolumn{5}{|l|}{ Automatic $^{b}$} \\
\hline$I_{\mathrm{dif}} \geq 1 \sigma$ & $1.73 \pm 0.05$ & $1.54 \pm 0.04$ & $1.77 \pm 0.05$ & $1.73-1.83$ \\
\hline$I_{\mathrm{dif}} \geq 2 \sigma$ & $1.71 \pm 0.06$ & $1.64 \pm 0.06$ & $1.77 \pm 0.06$ & $1.71-1.83$ \\
\hline$I_{\mathrm{dif}} \geq 3 \sigma$ & $1.73 \pm 0.08$ & $1.76 \pm 0.09$ & $1.79 \pm 0.08$ & $1.73-1.82$ \\
\hline
\end{tabular}

\footnotetext{
${ }^{a}$ The estimates of $\alpha$ for events identified by visual inspection.

${ }^{b}$ The estimates of $\alpha$ for events identified automatically, with the criteria $\Delta T_{p} \leq 3 \Delta t, L_{y} \geq 3$ pixels and $I_{\text {dif }} \geq 1 \sigma, 2 \sigma$, and $3 \sigma$.

${ }^{c}$ (Thermal Energy) fix $_{\text {axsumes }} L / L_{y}=3$. (Thermal Energy) random assumes $L / L_{y}=f$, where $f$ is chosen randomly in the range 2-7.
}

where $\mathrm{d} N$ is the number of events recorded with the parameter $x$ of interest between $x$ and $x+\mathrm{d} x$, and $A$ and $\alpha$ are constants which are most commonly determined from a least-squares fit to histograms of the data (e.g. Drake 1971; Lin et al. 1984; Hudson 1991; Crosby et al. 1993; Shimizu 1995; Veronig et al. 2002). The observed data usually show a drop-off in event numbers below the sensitivity threshold. This could result from the under-reporting of the true event energies or from the reduced efficiency of finding faint events. Parnell \& Jupp (2000) use a second power law to describe the drop-off in events. Thus there are three free parameters in the fit. For example, if the parameter $x$ of interest is taken as the energy, $E$, the frequency distribution is

$$
p(E)= \begin{cases}p_{0}(\alpha-1)\left(E / E_{0}\right)^{-\alpha} / E_{0} & \left(E \gtrsim E_{0}\right), \\ 0 & \left(E \lesssim E_{0}\right),\end{cases}
$$

but the observed event energies, $E_{\mathrm{obs}}$, will have a skew-Laplace frequency distribution of the form

$$
p_{\text {obs }}\left(E_{\text {obs }}\right)=\left\{\begin{array}{c}
p_{0}(\phi+1)(\alpha-1)\left(E_{\mathrm{obs}} / E_{0}\right)^{-\alpha} /\left[E_{0}(\phi+\alpha)\right] \\
\quad\left(E_{0} \lesssim E_{\mathrm{obs}}<\infty\right), \\
p_{0}(\phi+1)(\alpha-1)\left(E_{\mathrm{obs}} / E_{0}\right)^{\phi} /\left[E_{0}(\phi+\alpha)\right] \\
\quad\left(0<E_{\mathrm{obs}} \lesssim E_{0}\right)
\end{array}\right.
$$

where the fit parameters are $\alpha, \phi$ and $E_{0}$. In the following sections, we will apply the maximum likelihood method proposed by Parnell \& Jupp (2000) to calculate the power-law indices and threshold values for the observed frequency distributions of peak intensity, fluence and total energy.

\subsubsection{Frequency distributions of peak intensity and radiated energy}

The peak intensity frequency distributions for events identified automatically using the criteria $\Delta T_{p} \leq 3 \Delta t, L_{y} \geq 3$ pixels and $I_{\text {dif }} \geq 1 \sigma, 2 \sigma$, and $3 \sigma$ are shown in Fig. 8a, together with the results for the visual search (thick line circles). Our estimates of the threshold intensity, $I_{0}$, range from $5-9 \times 10^{-3} \mathrm{~W} \mathrm{~m}^{-2} \mathrm{sr}^{-1}$. Above the threshold all cases have a similar power-law index: 1.7 for the automatic searches and 1.6 for the visual search (Table 2). Below the threshold the visual search curve drops off much more rapidly for two reasons. Firstly, small events are missed, and secondly, big brightenings are not split into several individual events (Fig. 6). The plots of event frequency against fluence on the right (Fig. 8b), are more sensitive to the search method. The threshold value for the visual case is ten times that of the $1 \sigma$ case. The power-law indices beyond the threshold are 1.5-1.8 for the automatic searches and 1.8 for the visual. These are consistent with regular soft X-ray flares $(\alpha \approx 1.8)$ (e.g. Drake 1971) and microflares ( $\alpha=1.6-1.9)$ (e.g. Shimizu 1995). Another feature of these distributions is the gradual increase in the shortfall of events below the fitted power-law towards the highest event peak intensities and energies. We suspect that this is due to using the temperature specific Fe XIX line and possibly to our restricted field-of-view. The larger, brighter events probably raise the plasma temperature above $6.3 \mathrm{MK}$, so the Fe XIX captures the post-flare cooling not the event peak, leading to an underestimate in event energy. As mentioned previously, some of the larger events may be under-represented if the slit cuts the two legs of a loop or $L / L_{y}$ increases with event energy.

\subsubsection{Frequency distribution of thermal energy}

To estimate the thermal energy content of each event, we need to know the loop geometry; however, SXT images are available for only a few events so that most of the the loops can not be identified. As already mentioned in Sect. 4.2, larger loops generally produce brightenings with a larger extension along the slit. So we may simply assume that there is a linear scaling between the loop length, $L$, and the brightening extension, $L_{y}$, e.g., take $L / L_{y}=3$. Note that the exact value of this ratio does not change the estimated value of the power-law index $\alpha$, but it shifts the energy range and affects the estimate of the total power. If we further assume that the temperature of brightening loops is $6.3 \mathrm{MK}$ and the loop width, $w$, is $7 \mathrm{Mm}$, we can estimate the thermal energy by

$E_{\text {therm }}=3 n_{\mathrm{e}} k_{\mathrm{B}} T\left(L w^{2}\right)$,

where the electron density $n_{\mathrm{e}}$ is derived from the peak intensity mentioned in Sect. 4.2. We obtain thermal energies in the range $10^{26}-2 \times 10^{29} \mathrm{erg}$ (Table 1). This energy range is consistent with that obtained for SXT microflares (Shimizu 1995).

Figure 9a shows the fitted and empirical frequency functions of the thermal energy for the events which match the criteria of $\Delta T_{p} \leq 3 \Delta t, L_{y} \geq 3$ pixels and $I_{\mathrm{dif}} \geq 1 \sigma, 2 \sigma$, and $3 \sigma$. The threshold energy $E_{0}$ is estimated to be about $(1-2) \times 10^{27} \mathrm{erg}$ and the power-law index, $\alpha \approx 1.8$ (Table 2 ). For comparison, we also estimate $\alpha$ for the thermal energy distribution in the visual inspection case, giving a value of $2.05 \pm 0.15$. This estimate is larger than the critical value 2 (Hudson 1991), however with big uncertainties due to the small sample size.

In all the cases considered above, we have assumed that $L / L_{y}=3$. However, for a certain flaring loop the intersection length of the loop with the slit can vary when the slit intersects different parts of the loop (leg or top), or the loop is twisted with respect to the line-of-sight. So the ratio of the loop length to the brightening extension may take any value in a certain range. Let us consider this situation by assuming $L / L_{y}=f$, where $f$ is chosen randomly in the range $2-7$. The thermal energy frequency distributions obtained for 5 such random series give estimates of 

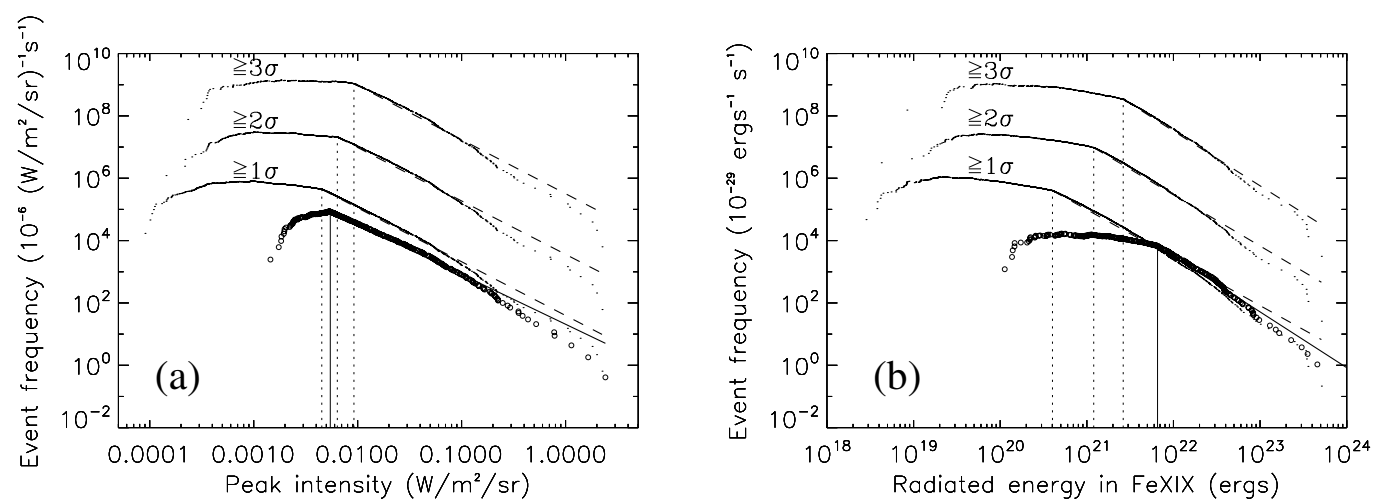

Fig. 8. Frequency of events vs. a) event peak intensity, and b) event integrated energy in the Fe XIX line for events of intensity enhancements at least $1 \sigma, 2 \sigma$, and $3 \sigma$. These plots show the observed data (solid line and dots) and the right-hand power law of the fitted skew-Laplace distribution (dashed line). The frequencies of events for the three cases in a) and b) are multiplied by $1,10^{2}, 10^{4}$, respectively, so that they can all be drawn without overlap on the same graph. For comparison, the data (circles) obtained from the visual inspection case are also overlaid. The vertical lines (dashed and solid lines) mark estimates of the threshold intensity in a) and radiated energy in b).
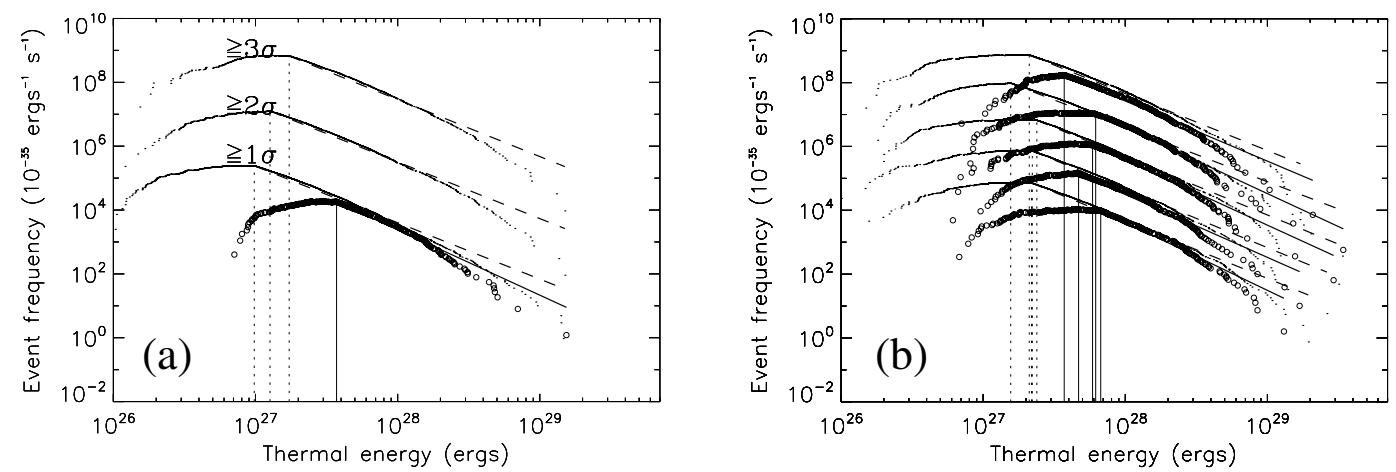

Fig. 9. Frequency of events vs. event thermal energy. a) The thermal energy of an event is calculated by assuming the loop length to be three times the brightening extension along the slit (See main text for details). The frequencies of events for the three cases (with intensity enhancements at least $1 \sigma, 2 \sigma$, and $3 \sigma$ ) are multiplied by $1,10^{2}, 10^{4}$, respectively. b) The thermal energy of an event is calculated by assuming the loop length to be the brightening extension along the slit times a uniformly-distributed random factor in the range $2-7$. The frequencies of events (with intensity enhancements at least $2 \sigma$ ) for the 5 cases (corresponding to 5 independent random series) are multiplied by $1,10,10^{2}, 10^{3}, 10^{4}$, respectively. The data (circles) obtained from the visual inspection case are also overlaid. The vertical lines (dashed and solid lines) mark estimates of the threshold intensity in thermal energy.

$\alpha$ consistent with the result obtained for the case of $L / L_{y}=3$ (Table 2). This test shows that the exact values of $L / L_{y}$ are not essential for determining $\alpha$. We have also considered other relationships between $L$ and $L_{y}$. For example, the scaling $L \sim L_{y}^{2}$ which represents cases where the slit intersects loop tops (Wang et al. 2003a). This stretches the energy range and thus reduces the $\alpha$. For the automatic search results we computed $\alpha=1.5$, giving a $15 \%$ reduction in $\alpha$ compared with the $L / L_{y}=3$ scaling.

\subsubsection{Estimates of total energy input}

The result of $\alpha \sim 1.8$ for the frequency distribution of thermal energy is consistent with estimates of the microflare power-law index and supports the idea that the Fe XIX brightenings are the coronal parts of microflares. In this section, we estimate how much energy the observed Fe XIX flarelike events can contribute based on their occurrence rate. We can estimate the averaged observed total power, $P\left(E_{\mathrm{obs}}\right)$, as the thermal energy of all events divided by the total observation time, $\Delta T$. For the two data sets analysed, $\Delta T=96.6 \mathrm{~h}$ and the total number of events, $N=1334$, 863,633 for events matching $I_{\text {dif }} \geq 1 \sigma, 2 \sigma, 3 \sigma, \Delta T_{p} \leq 3 \Delta t$ and $L_{y} \geq 3$ pixels. So the average observed event occurrence rate, $p_{0}$, is $7-14$ per hour. The event rate for the visual search is 3 per hour, but the average event energy is double (Table 1), and therefore the difference in the estimates of total power obtained with the two search methods is at most a factor 3 . We obtain that $P\left(E_{\text {obs }}\right) \approx(7-20) \times 10^{24} \mathrm{erg} \mathrm{s}^{-1}$.

We can compare this with estimates assuming a power law distribution of events, where the total power can be represented by,

$P=\int_{E_{\min }}^{E_{\max }} f(E) E \mathrm{~d} E$.

$f(E)$ is the event energy frequency distribution function with dimensions of $\mathrm{erg}^{-1} \mathrm{~s}^{-1}$ and the limits $E_{\min }$ and $E_{\max }$ are the energies of the smallest and largest events, respectively. Using Eq. (2), we have

$P=p_{0} E_{0} \frac{\alpha-1}{2-\alpha}\left[\left(\frac{E_{\max }}{E_{0}}\right)^{2-\alpha}-\left(\frac{E_{\min }}{E_{0}}\right)^{2-\alpha}\right]$,

where $E_{0}$ is the threshold energy and $p_{0}$, derived from

$\frac{N}{\Delta T}=\int_{E_{\min }}^{E_{\max }} f(E) \mathrm{d} E$, 
Table 3. Estimates of total power for events which match the criteria $I_{\text {dif }} \geq \mathcal{N} \sigma, \Delta T_{p} \leq 3 \Delta t$ and $L_{y} \geq 3$ pixels.

\begin{tabular}{cccccccc}
\hline \hline $\mathcal{N} \sigma$ & $\alpha$ & $\begin{array}{c}E_{0} \\
\left(10^{27} \mathrm{erg}\right)\end{array}$ & $\begin{array}{c}E_{\min } \\
\left(10^{26} \mathrm{erg}\right)\end{array}$ & $\begin{array}{c}E_{\max } \\
\left(10^{29} \mathrm{erg}\right)\end{array}$ & $\begin{array}{c}P\left(E_{\mathrm{obs}}\right) \\
\left(10^{25} \mathrm{erg} / \mathrm{s}\right)\end{array}$ & $\begin{array}{c}P\left(E_{0}: E_{\max }\right) \\
\left(10^{25} \mathrm{erg} / \mathrm{s}\right)\end{array}$ & $\begin{array}{c}P\left(E_{\min }: E_{\max }\right) \\
\left(10^{25} \mathrm{erg} / \mathrm{s}\right)\end{array}$ \\
\hline $1 \sigma$ & 1.77 & 1.0 & 1.0 & 1.5 & 1.8 & 2.8 & 2.1 \\
$2 \sigma$ & 1.77 & 1.3 & 1.2 & 1.5 & 1.4 & 2.1 & 1.7 \\
$3 \sigma$ & 1.79 & 1.7 & 1.5 & 1.5 & 1.3 & 1.9 & 1.5 \\
\hline
\end{tabular}

Note: $\mathcal{N} \sigma$ is the noise level of the intensity. $\alpha$ is the power-law index of the frequency distribution of thermal energy. $E_{0}$ is the threshold thermal energy. $E_{\min }$ and $E_{\max }$ are the minimum and maximum thermal energies for events, respectively. $P\left(E_{\mathrm{obs}}\right)$ is the total power estimated by the total thermal energies of all detected events divided by the whole duration of the data. $P\left(E_{0}: E_{\max }\right)$ is the total power estimated by Eq. (6) for events with $E_{\min }=E_{0} . P\left(E_{\min }: E_{\max }\right)$ is the total power estimated by Eq. (6).

is given by

$p_{0}=\frac{N}{\Delta T\left[\left(E_{\min } / E_{0}\right)^{1-\alpha}-\left(E_{\max } / E_{0}\right)^{1-\alpha}\right]}$.

The choice for $E_{\min }$ is discussed in Parnell \& Jupp (2000). There are two choices. We can either take $E_{\min }=E_{0}$ or $E_{\min }=$ $E_{\text {min }}$ (observed). If we consider the case where the observed event frequency drop-off is due to under-estimating the true event energy, then $E_{\min }=E_{0}$ in Eqs. (6) and (8) and $N$ is the total number of observed events. If the drop-off is caused by underreporting of small events, then $p_{0}$ is computed with $E_{\min }=E_{0}$, $N$ is the number of observed events above $E_{0}$, and in the estimate of total power (Eq. (6)) $E_{\min }=E_{\min }$ (observed). In Table 3, we give the energy estimates for both cases. The power is about $50 \%$ more than $P\left(E_{\mathrm{obs}}\right)$ for the case when the energy drop-off is due to under-estimating the energy. This gives an idea of how much energy is lost in the background emission.

The amount of energy in the background can also be estimated from the observations directly. By averaging the total emission over the whole slit during the entire observing period, we obtain a mean intensity of $8.0 \mathrm{erg} \mathrm{cm}-2 \mathrm{~s}^{-1} \mathrm{sr}^{-1}$ from the original data in the Fe XIX line and of $5.4 \mathrm{erg} \mathrm{cm}^{-2} \mathrm{~s}^{-1} \mathrm{sr}^{-1}$ for the data with the background emission removed. If the total emission from events matching $I_{\text {dif }} \geq 1 \sigma, \Delta T_{p} \leq 3 \Delta t$ and $L_{y} \geq 3$ pixels is averaged in the same way, we obtain a mean intensity of $3.3 \mathrm{erg} \mathrm{cm}^{-2} \mathrm{~s}^{-1} \mathrm{sr}^{-1}$. Thus we find that the average background intensity is comparable to that of the identified events and more than an order of magnitude larger than the peak intensity of the smallest event (see Table 1).

\section{Doppler shifts}

Many of the Fe XIX brightenings are associated with Doppler shift oscillations. This phenomenon has been studied in detail in a series of papers by Wang et al. (2002, 2003a,b, 2005). The observed Doppler shift oscillations have been interpreted as standing slow mode waves (Ofman \& Wang 2002; Wang et al. 2003a). For 415 brightenings, identified from the first data set with the criteria $I_{\text {dif }} \geq 2 \sigma, \Delta T_{p} \leq 3 \Delta t$, and $L_{y} \geq 3$ pixels, we find that brightenings have an average maximum Doppler shift of $24 \pm 37 \mathrm{~km} \mathrm{~s}^{-1}$. The Doppler shifts cover a very broad range $3-284 \mathrm{~km} \mathrm{~s}^{-1}$, but most of them $(\sim 97 \%)$ have maximum shifts below $100 \mathrm{~km} \mathrm{~s}^{-1}$. The size of the shifts may be underestimated because we have employed a single Gaussian fit to the line profiles, and in many cases there may be a weak highly shifted component (Wang et al. 2005). The initial Doppler shifts are most likely caused by a pulse of hot plasma travelling along the loop, which implies that the released energy in the event not only heats the plasma to a temperature of at least $6 \mathrm{MK}$ (formation temperature of Fe XIX), but also accelerates the plasma. Let us consider the ratio of the kinetic energy to the thermal energy in such events,

$$
\frac{\text { Kinetic Energy }}{\text { Thermal Energy }}=\frac{(1 / 2) m V^{2}}{(3 / 2) k_{\mathrm{B}} T}=0.04 \frac{\left(V / 100 \mathrm{~km} \mathrm{~s}^{-1}\right)^{2}}{(T / 6 \mathrm{MK})} \text {, }
$$

where $k_{\mathrm{B}}$ is the Boltzmann constant, $V$ and $T$ are the maximum velocity and temperature of the heated and accelerated plasma. Finally, $m$ is the mean particle mass, taken here to be $0.6 m_{p}$ (assuming a standard coronal He abundance), where $m_{p}$ is the mass of a proton. Because most events have their maximum Doppler velocities below $100 \mathrm{~km} \mathrm{~s}^{-1}$, the kinetic energy of events is at least a factor of 25 less than the thermal energy, and an insignificant energy source for the corona.

\section{Summary and discussions}

In active region coronae, we find a large number of small coronal brightenings in the Fe XIX line. At a height of about $30 \mathrm{Mm}$ there are on average 3-14 brightenings per hour from the active regions analysed. In this first analysis of our data, we have looked at the statistics of the events and find that the measured event frequency, lifetimes and sizes suggest they represent emission from loops excited by soft X-ray microflares (e.g. Drake 1971; Shimizu 1995). Relatively intense events are associated with GOES C-class flares. Many of these brightenings are associated with Doppler shift oscillations, which have been interpreted as slow mode standing waves in hot coronal loops (e.g. Wang et al. 2003b; Innes \& Wang 2004).

For events with intensity enhancements of at least $1 \sigma, 2 \sigma$, and $3 \sigma$, estimates of $\alpha$ are 1.7-1.8 for the thermal energy distribution above $10^{27} \mathrm{erg}$, in accord with active region soft $\mathrm{X}$ ray brightenings. The drop-off in events with low energies could have two reasons. One could be because the slit is fixed at $30 \mathrm{Mm}$ off the limb, so that events in loops shorter than about $60 \mathrm{Mm}$ will be below the slit. The other is due to the strong background which may obscure faint events. Modelling by Pauluhn \& Solanki (2006) suggests that if the background is explicitly included in the analysis then larger values of $\alpha$ can be obtained. If $\alpha$ is greater than 2, the contribution of the small events to the overall energy release may be significant.

With the measured event occurrence rate and $\alpha$, we estimate the total power contributed by the flarelike brightenings seen in Fe XIX. For the power-law distribution and energy range from $10^{26}$ to $2 \times 10^{29} \mathrm{erg}$, the total power is about $(2-3) \times 10^{25} \mathrm{erg} \mathrm{s}^{-1}$. If we further consider that the analysed active regions have a size of about $150^{\prime \prime}$ (i.e. $L_{\mathrm{AR}} \sim 100 \mathrm{Mm}$ ) (see Fig. 1), then the total power per unit area, given by $P\left(E_{\min }: E_{\max }\right) / L_{\mathrm{AR}}^{2}$, is about $2 \times 10^{5} \mathrm{erg} \mathrm{cm}^{-2} \mathrm{~s}^{-1}$. This is not sufficient for the heating of the active-region corona, which has a typical energy loss rate of about $10^{7} \mathrm{erg} \mathrm{cm}^{-2} \mathrm{~s}^{-1}$ (Withbroe \& Noyes 1977; Aschwanden 2004). However, this is not the total active region 
energy in Fe XIX loops because not all events reach the height of the SUMER slit. If we consider the microflare loop distribution observed by Shimizu et al. (1994) then only $10 \%$ of events occur in loops longer than $50 \mathrm{Mm}$ and thus reach the slit. Nevertheless the events that do are likely to be the bigger, more energetic ones which we suspect dominate the energy. It will be an interesting future observation to measure flarelike brightenings down to the limb and obtain a more complete estimate of the energy input.

Acknowledgements. SUMER is financially supported by DLR, CNES, NASA and the ESA PRODEX programme (Swiss contribution). SOHO is a project of international co-operation between ESA and NASA.

\section{References}

Aschwanden M. J. 2004, in SOHO 15 Coronal Heating, ESA SP-575: ed. R. W. Walsh, J. Ireland, D. Danesy, \& B. Fleck, 97

Berghmans, D., McKenzie, D., \& Clette, F. 2001, A\&A, 369, 291

Brković, A., Solanki, S. K., \& Rüedi, I. 2001, A\&A, 373, 1056

Crosby, N., Aschwanden, M., \& Dennis, B. 1993, Adv. Space Res., 13, 179

Drake, J. F. 1971, Sol. Phys., 16, 152

Feldman, U., Landi, E., Doschek, G. A., Dammasch, I., \& Curdt, W. 2003, ApJ, 593, 1226
Gary, D. E., Hartl, M. D., \& Shimizu, T. 1997, ApJ, 477, 958

Hudson, H. S. 1991, Sol. Phys., 133, 357

Innes, D. E., \& Wang, T. J. 2004, in ESA SP-575: SOHO 15 Coronal Heating, 553

Lin, R. P., Schwartz, R. A., Kane, S. R., Pelling, R. M., \& Hurley, K. C. 1984, ApJ, 283, 421

Nitta, N. 1997, ApJ, 491, 402

Ofman, L., \& Wang, T. 2002, ApJ, 580, L85

Parnell, C. E., \& Jupp, P. E. 2000, Astrophysics, 529, 554

Pauluhn, A., \& Solanki, S. K. 2006, A\&A, submitted

Qiu, J., Liu, C., Gary, D. E., Nita, G. M., \& Wang, H. 2004, ApJ, 612, 530

Shimizu, T. 1995, PASJ, 47, 251

Shimizu, T., Tsuneta, S., Acton, L. W., Lemen, J. R., Uchida, Y. 1992, PASJ, 44, L147

Shimizu, T., Tsuneta, S., Acton, L. W., et al. 1994, ApJ, 422, 906

Tsuneta, S., Acton, L., Bruner, M., et al. 1991, Sol. Phys., 136, 37

Veronig, A., Temmer, M., Hanslmeier, A., Otruba, W., \& Messerotti, M. 2002, A\&A, 382, 1070

Wang, T., Solanki, S. K., Curdt, W., Innes, D. E., \& Dammasch, I. E. 2002, ApJ, 574, L101

Wang, T. J., Solanki, S. K., Curdt, W., et al. 2003a, A\&A, 406, 1105

Wang, T. J., Solanki, S. K., Innes, D. E., Curdt, W., \& Marsch, E. 2003b, A\&A, 402, L17

Wang, T. J., Solanki, S. K., Innes, D. E., \& Curdt, W. 2005, A\&A, 435, 753

Withbroe, G. L., \& Noyes, R. W. 1977, ARA\&A, 15, 363

Young, P. R., Del Zanna, G., Landi, E., et al. 2003, ApJS, 144, 135 